\title{
Heat transfer in fuel oil storage tank at thermal power plants with local fuel heating
}

\author{
Svetlana A. Kuznetsova and Vyacheslav I. Maksimov \\ Institute of Power Engineering, National research Tomsk Polytechnic University, Tomsk 634050, Russia
}

\begin{abstract}
Results of mathematical modeling of the thermal control system in fuel oil storage, in the presence of heat source at the lower boundary of the region, in the framework of models of incompressible viscous fluid are presented. Solved the system of differential equations of non-stationary Navier-Stokes equations, the energy equation and the heat equation with appropriate initial and boundary conditions. Takes into account the processes of heat exchange region considered with the environment. A comparative analysis of the dependence of average temperatures of oil in the volume of the tank on the time calculated by the simplified (balanced) method and obtained as a result of numerical simulation are performed.
\end{abstract}

\section{Introduction}

The main problems of use (handling, storage, supply) fuel oil at thermal power plants in the colder periods of the year are related with the dependence of the viscosity of this fuel on temperature [1]. In the preparation of fuel oil for burning in the winter, as a rule, expended a lot of energy on its heated to a temperature corresponding to the procedural regimes. Until now the choice of process parameters and schemes of heating such liquid fuel is mainly conducted using relatively simple models [2], do not take into account possible spatial character of the temperature distribution in the conditions of local heating the large volumes of this liquid fuel. At the same time, probably may be reduced the energy consumption for heating oil through the optimal choice of scheme and conditions of the heat supply in fuel oil storage tank. But such choice is difficult on the results of experimental studies. It is expedient to modeling the thermal control system on fuel oil storage tank with the use of systems of equations describing the processes of heat conduction and convection occurring in the system "liquid oil - a source of heat - fuel oil storage wall".

In physics under study process the considered problem has much in common with the problem of free convection in a cavity with conducting walls and the local energy source [3, 4]. A feature of heat and mass transfer at movement oil are its high density (compared to air [2]) and the dependence of viscosity on temperature. Within the model of incompressible viscous fluid heating regimes on fuel oil storage tank is practically not been studied until recently.

Purpose of work - analysis of non-stationary thermal fuel oil storage mode using a heat transfer model that takes into account the thermal conductivity, natural convection, the local supply of energy and the heat sink through heat conduction and heat storage walls.

\section{Statement of the problem}

It has been considered the fuel oil storage tank (Figure 1) of rectangular shape with horizontal and vertical walls of finite thickness and a heat source with a given surface temperature at the bottom edge region. At the initial time the temperature of the liquid fuel is greater than the initial temperature of the tank walls and the environment. It is assumed that at the boundaries of "liquid - solid wall" the condition of perfect contact, at the external borders the conditions of heat dissipation into the environment.

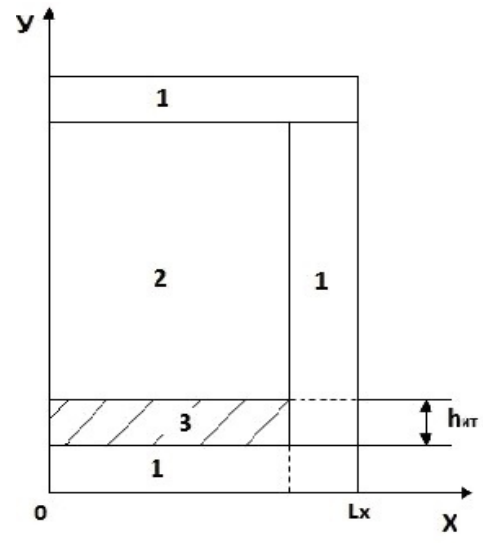

Figure 1. Area of solution the problem. 
Formulation of the problem in the spatial coordinates is described by a system of two-dimensional and non-stationary NavierStokes equations and the energy equation for the liquid phase (oil) and heat conduction equation for reinforced concrete wall of the tank, by analogy with $[3,4]$.

$$
\begin{gathered}
\frac{\partial \Omega}{\partial \tau}+U \frac{\partial \Omega}{\partial X}+V \frac{\partial \Omega}{\partial Y}=\frac{1}{\operatorname{Re}}\left(\frac{\partial^{2} \Omega}{\partial X^{2}}+\frac{\partial^{2} \Omega}{\partial Y^{2}}\right)+\frac{G r}{\operatorname{Re}^{2}}\left(\frac{\partial \Theta}{\partial X}\right), \\
\frac{\partial \Theta}{\partial \tau}+U \frac{\partial \Theta}{\partial X}+V \frac{\partial \Theta}{\partial Y}=\frac{1}{\operatorname{Re} \cdot \operatorname{Pr}}\left(\frac{\partial^{2} \Theta}{\partial X^{2}}+\frac{\partial^{2} \Theta}{\partial Y^{2}}\right), \\
\frac{\partial^{2} \Psi}{\partial X^{2}}+\frac{\partial^{2} \Psi}{\partial Y^{2}}=\Omega, \\
\frac{1}{F o} \frac{\partial \Theta}{\partial \tau}=\frac{\partial^{2} \Theta}{\partial X^{2}}+\frac{\partial^{2} \Theta}{\partial Y^{2}},
\end{gathered}
$$

here

$G r=\frac{g \beta L^{3} \Delta T}{v^{2}}-$ Grashof number;

$\beta$ - thermal coefficient of volume expansion; $g$ - acceleration created by the mass forces; $v$ - coefficient of kinematic viscosity of the fluid; Re - Reynolds number; $\mathrm{Pr}$ - Prandtl number; $\mathrm{F}_{0}$ - Fourier number.

The initial conditions for the system of equations:

$$
\Psi(X, Y, 0)=0, \Omega(X, Y, 0)=0, \Theta(X, Y, 0)=0 .
$$

Boundary conditions:

- on the outer contour of considered area

$$
\mid \begin{aligned}
& Y=0,0 \leq X \leq \frac{l_{1}+l_{2}+l_{u m}}{L} \\
& X=\frac{l_{1}+l_{2}+l_{u m}}{L}, 0 \leq Y \leq \frac{h_{1}+h_{2}+H}{L} \\
& Y=\frac{h_{1}+h_{2}+H}{L}, 0 \leq X \leq \frac{l_{1}+l_{2}+l_{u m}}{L}
\end{aligned}
$$

are set the boundary conditions of the second kind $\frac{\partial \Theta}{\partial n}=K i$;

- on the axis of symmetry $X=0$ conditions of the form

$$
\frac{\partial \Theta}{\partial X}=\Omega=\Psi=0
$$

- at the internal borders between the solid and liquid phase, parallel to the axis $0 \mathrm{Y}$ accepted:

$$
\mid \begin{aligned}
& \Psi=0, \frac{\partial \Psi}{\partial X}=0, \\
& \left\{\begin{array}{l}
\Theta_{w}=\Theta_{f}, \\
\frac{\partial \Theta_{w}}{\partial X}=\lambda_{w, f} \frac{\partial \Theta_{f}}{\partial X},
\end{array} \quad \text { at } X=\frac{l_{1}}{L}, \frac{h_{1}}{L} \leq Y \leq \frac{h_{1}+H}{L}\right.
\end{aligned}
$$

- at the internal borders between the solid and liquid phase, parallel to the axis $0 \mathrm{X}$ accepted:

$$
\left\{\begin{array}{l}
\Psi=0, \frac{\partial \Psi}{\partial Y}=0, \\
\left\{\begin{array}{l}
\Theta_{w}=\Theta_{f}, \\
\frac{\partial \Theta_{w}}{\partial Y}=\lambda_{w, f} \frac{\partial \Theta_{f}}{\partial Y},
\end{array} \text { at } Y=\frac{h_{2}}{L}, \frac{l_{1}}{L} \leq X \leq \frac{l_{1}+l_{u m}}{L}\right.
\end{array}\right.
$$

- On the local heat source are specified boundary conditions of the second kind:

$$
\frac{\partial \Theta}{\partial n}=K i
$$

here $K i=\frac{q L}{\lambda_{w}\left(T_{i t}-T_{0}\right)}-$ Kirpichev number; $\lambda_{w}$ - coefficient of thermal conductivity of the solid phase; $\lambda_{f}-$ coefficient of thermal conductivity of the liquid phase; $\lambda_{w, f}=\lambda_{w} / \lambda_{f}$ - relative coefficient of thermal conductivity; $q-$ heat flow on the outer boundary solutions. 
The boundary value problem (1-4) with appropriate boundary and initial conditions was solved by finite difference method using the algorithm $[5,6]$ that is designed to the numerical solution of nonlinear problems of heat and mass transfer with inhomogeneous boundary conditions.

Equations were solved sequentially; each time step begins with the calculation of the temperature field in the liquid and solid phases, and then solved the Poisson equation (3) for the vector potential. Then was determined the boundary conditions for vector components of the vorticity and solved the equations of motion.

\section{Analysis of the numerical modeling results}

Numerical studies have been conducted with the following values: $T_{0}=293 \mathrm{~K}, 253 \leq T_{e} \leq 293 \mathrm{~K}, 100 \leq q \leq 150 \mathrm{~kW} / \mathrm{m}^{2}$.

Ranges of variation of size characteristics were chosen from the condition of compliance with range of parameter changes in real-world implementations of such systems.

Fig. 2 - 3 shows typical results of numerical investigations as isolines of the stream function and temperature fields.
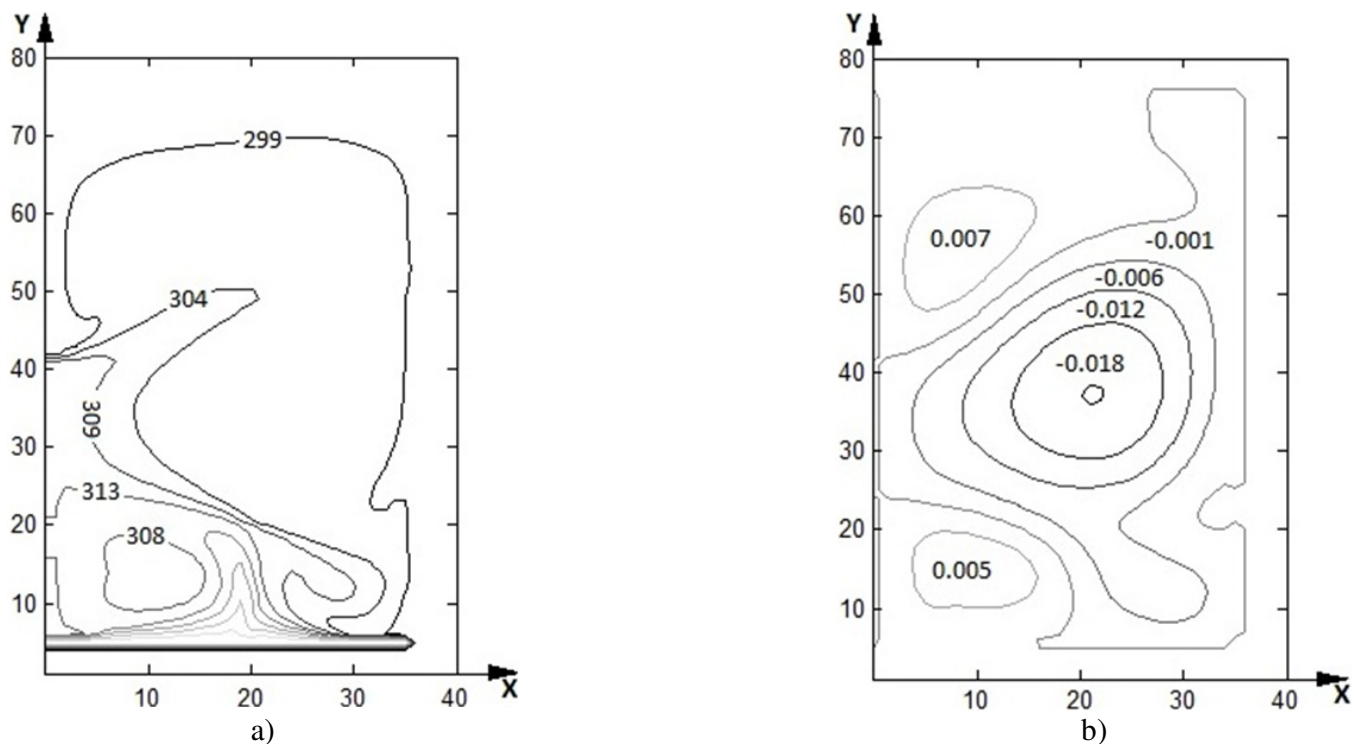

Figure 2. Temperature distribution (a) and contours of the stream function (b) at $\mathrm{q}=100 \mathrm{~kW} / \mathrm{m}^{2}$
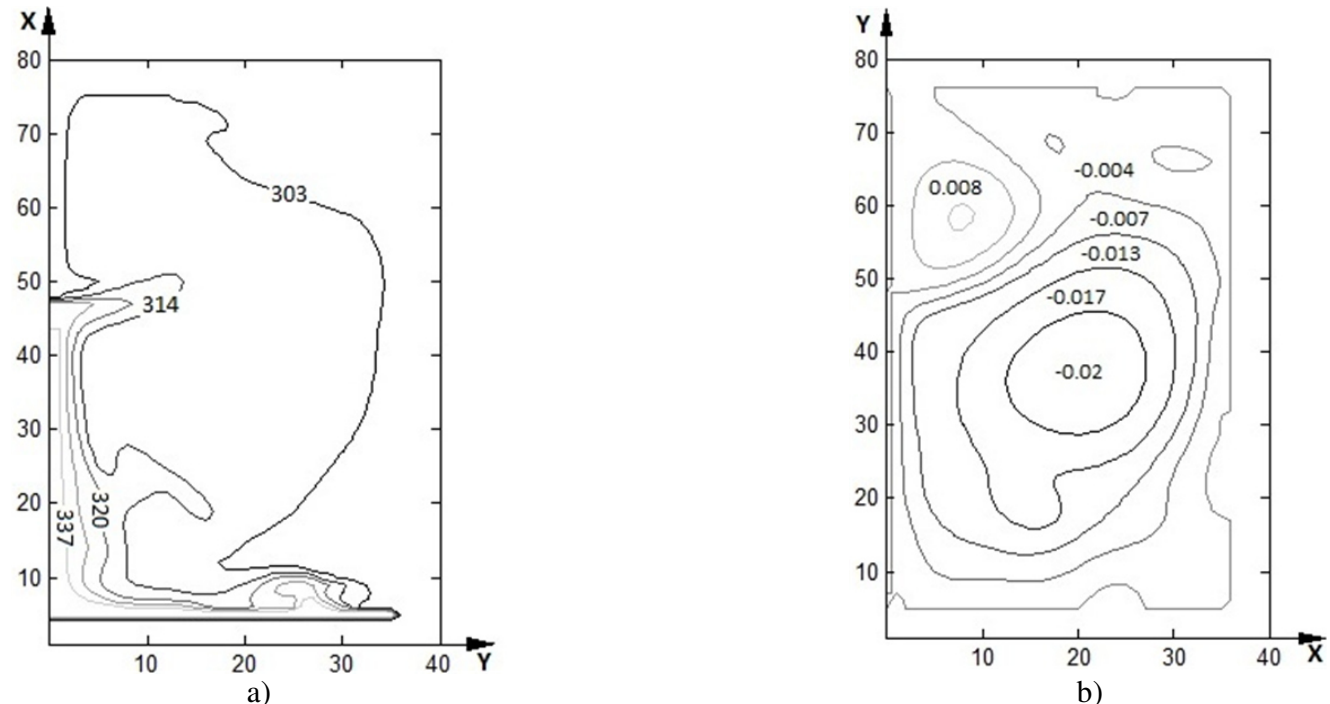

Figure 3. Temperature distribution (a) and contours of the stream function (b) at $\mathrm{q}=150 \mathrm{~kW} / \mathrm{m}^{2}$

Figure 2 clearly shows the formation of three vortices in the central part of the tank as a result of natural convection when the heat flux on the heat source $\mathrm{q}=100 \mathrm{~kW} / \mathrm{m}^{2}$. This character of vortex formation is due to the heat sink on the vertical boundary of the tank (the problem is solved in an axisymmetric formulation). The bulk of oil during heating process is raised to the top of the tank near its axis of symmetry, and cooled (by heat exchange on the vertical outer boundary of the solution) fuel is moved down. In this case clearly shows that, for example, during the time of warm-up about 3600s oil temperature field is not homogeneous (there are quite significant temperature gradients). Thus the maximum temperature to which the oil has time to warm up under given conditions, it is $\mathrm{T}_{\max }=320 \mathrm{~K}$, while the minimum temperature is equal to $\mathrm{T}_{\min }=295 \mathrm{~K}$.

Increasing the intensity of the energy supply at the lower boundary (Fig.3.) to $150 \mathrm{~kW} / \mathrm{m}^{2}$ leads to a significant changes in both the hydrodynamics of flow and temperature fields. As a consequence becomes significantly more intense the vortex formation in the central part of the tank and accordingly oil is warmed up to high values of temperature. 
Also worth noting the quite obvious asymmetry of the temperature field (Figure 3.), which is due to the heat sink on the outer vertical boundary of the tank. In this case, the maximum temperature of the heated oil during the accounting period of time will be $\mathrm{T}_{\max }=337 \mathrm{~K}$, and the minimum will be equal to $\mathrm{T}_{\min }=299 \mathrm{~K}$.

In order to justify the advisability of determining the temperature fields on the results of the solution of the problem (1) - (12) were calculated the average temperature of oil in the volume of the tank. Also, average temperatures of oil were calculated by simplified (balanced) method [2]. Comparing the obtained results shown in Fig. 4.
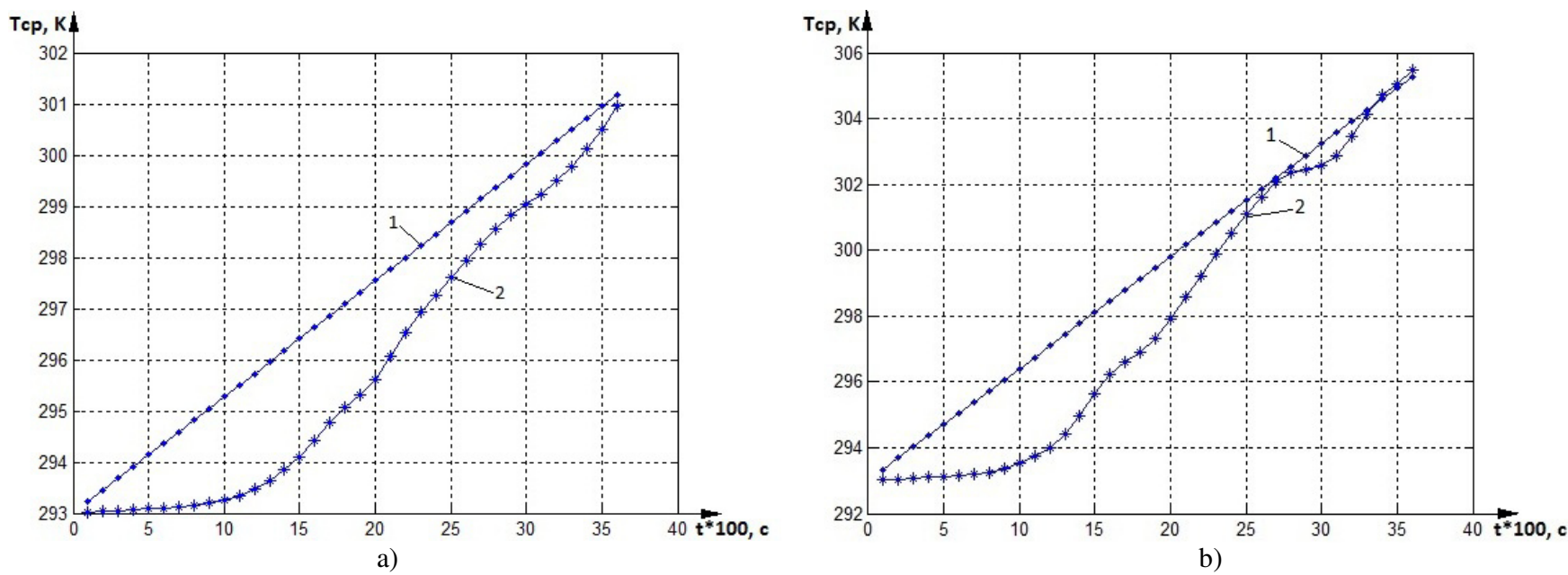

Figure 4. The dependences of the average temperature of oil on the time calculated by the simplified (balanced) method (1) and the obtained from numerical simulation (2). The heat flow is equal accordingly: a) $\mathrm{q}=100 \mathrm{~kW} / \mathrm{m}^{2}$; $\mathrm{b}$ ) $\mathrm{q}=150 \mathrm{~kW} / \mathrm{m}^{2}$.

Analyzing the dependence shown in Fig. 4 can be drawing a few conclusions. First, irrespective of magnitude of the heat flux at the lower boundary of the solutions, the largest deviations in the average temperature of fuel in the entire volume of the tank take place at the initial stage of heating. Secondly, it is clear that with increasing of heat supply intensity at the lower boundary of the tank oil temperature the deviation of averages calculated by simplified method and obtained from numerical simulation at the initial stage of heating increases. For example, in the time about $1000 \mathrm{~s}$ and when the value of heat flux $\mathrm{q}=100 \mathrm{~kW} / \mathrm{m}^{2}$, this deviation is $2 \mathrm{~K}$, while for $\mathrm{q}=150 \mathrm{~kW} / \mathrm{m}^{2}$ for $3 \mathrm{~K}$. However, it should be noted that by increasing the intensity of heat flux the average temperature value of oil calculated during the numerical simulations at large times slightly different from the average temperature obtained by balanced method. Also, comparison of the minimum temperature of oil in the tank with the mean temperature of the entire volume of fuel at the final time for different values of the heating intensity can be seen that the difference between them varies within 6-6.5 $\mathrm{K}$.

\section{Conclusion}

It is established that the value deviations of the average temperature of fuel in all storage volume calculated by simplified (balanced) method and obtained by numerical simulation results, can be considered negligible. This is constitute grounds for conclusion about the possibility of investigation of energy efficiency technologies for regulated heating modes of fuel oil storage within the framework of models of incompressible viscous fluid.

\section{References}

1. ECPs and economy of Russia regions. Reference: V.7. - Moscow: Energy. (2007)

2. Yu.G. Nazmeev, Fuel oil farms at thermal power plant. - Moscow: Publishing House of the Moscow Power Engineering Institute, 2002

3. G.V. Kuznetsov, M.A. Sheremet, Two-dimensional problem of natural convection in a rectangular domain with local heating and heat-conducting boundaries of finite thickness, Fluid Dynamics. V. 41. No. 6. (2006) 881-890

4. G.V. Kuznetsov, M.A. Sheremet, New approach to the mathematical modeling of thermal regimes for electronic equipment, Russian Microelectronics. V. 37. No. 2. (2008) 131-138

5. G.V. Kuznetsov, M.A. Sheremet, Mathematical modelling of complex heat transfer in a rectangular enclosure, Thermophysics and Aeromechanics. V. 16. No. 1. (2009) 119-128

6. V.M. Paskonov, V.I. Polezhaev, L.A. Chudov, Numerical modeling of heat and mass transfer. - Moscow: Nauka, 1984 $288 \mathrm{p}$. 